\title{
On Publishing Research: A Paper is as Good as the Findings
}

\author{
Santosh K. Chaturvedi
}

Published online: 9 July 2021

(C) The Author(s), under exclusive licence to Springer Nature India Private Limited 2021

Any research should culminate in a suitable publication. The publication is a method of sharing one's thoughts, views, data, results and scientific knowledge with the scientific world. Thus, any publication is a worthy publication. However, many opinions exist about the nature and quality of publications.

It is noted that professionals give undue importance to high impact factor journals. The high impact value is an artificial score and is based on number of citations of articles in any particular journal. This can be judiciously manipulated by writing about controversial or topical matters. Review articles also get higher citation in other journals. Citations also depend on conclusions and implications of the study. Articles which are critical of other literature and those published in the initial months of a year have a higher chance of being cited. Also, articles which have methodological limitations are cited to point out these shortcomings! So not all citations reflect positive and valuable information.

Articles published in journals with highest impact factors are critically evaluated and shred to pieces in any research forum or journal club by junior postgraduates. Thus insisting on publication in high impact journals only is a misguided view point. There are

S. K. Chaturvedi $(\bowtie)$

Rehabilitation Committee, National Institute of Mental Health and Neurosciences, Bengaluru, India

e-mail: skchatur@gmail.com many new journals (not predatory) which have not yet established their credibility which also need to be encouraged. In such journals the publication should be valued on its own merit. The quality of paper should be determined by research stringency and its value as well as implications and not on the quality or impact factor of the journal. Even peer review can be biased and it varies from peer to peer. There are no absolute standards for peer review. Both blind and non-blind reviews have their own advantages and drawbacks. Recently, fraud has been noticed in peer reviewing, with authors connecting with potential reviewers for a favour, or doing the peer review on their behalf. Technology however, can detect this, with an extra effort. Editors have to be cautious of this unhealthy practice.

Thus, it would be ethical to judge any publication based on its own merit and not be misled by the citations or reputation of the journal. In this competitive world many open access journals are charging exorbitant prices making a common researcher to struggle to find a place for their publication. This misdirected focusing on certain journals and ignoring the value of other newer journals should be stopped. All publications should be viewed equally without discrimination to maintain justice and acknowledge their worth in the scientific world. There is no such thing as a perfect paper of publication. It is sad to see that papers which have no drawbacks are rejected by journals due to lack of space! Sometime one wonders 
if there should be a journal for such orphan papers, or papers rejected, not due to any flaw in the paper, but due to too many papers being submitted to a particular journal, which cannot accommodate such articles. In the end, the paper is as good as the knowledge it imparts.

The Journal of Psychosocial Rehabilitation and Mental Health has gone through its steady development and reached the current position and popularity. It maintains a fair peer review process and till now has not rejected any paper, recommended by peer reviewers, due to a lack of space in the journal. The contributions of authors, reviewers, editorial and publishing staff need to be acknowledged.

The current issue has interesting articles, of a wide variety, submitted from different regions of the world. Subtle cognitive impairment is noted in patients with chronic schizophrenia; however, a cognitive enhancement therapy has been discussed by the team from Canada and the US. in this issue. Stigma is noticed mainly towards severe mental illness like schizophrenia; however, Grover and colleagues discuss stigma felt by caregivers of patients with obsessive compulsive disorders. Other interesting articles are on the relationship between quality of life and social support among patients with schizophrenia and bipolar disorder, qualitative exploration with self-injuring youth in India and inter-rater reliability and development of capacity disorders over the course of a vocational training program. Other articles of contemporary importance included in this issue include one on responding to the global pandemic: a pulse of the wellbeing of clubhouse communities moving virtual; social rehabilitation through a community-based rehabilitation lens: empowerment, participation and inclusion of the elderly long-term unemployed in the re-employment process; the provision of preventive care for chronic disease risk behaviours to people with a mental health condition: a survey of staff from one Australian community, and lastly one on homeless persons with mental illness residing in welfare homes in Kerala, India. Finally, there is an editorial with a fresh perspective that all is not negative about negative symptoms in schizophrenia. Like in previous issues, the journal has published submissions made from USA, Australia, Germany, Finland, Canada, and different parts of India. 